\title{
Face Detection using Combined Skin Color Detector and Template Matching Method
}

\author{
Smita Tripathi \\ SOIT, RGPV Bhopal
}

\author{
Varsha Sharma \\ SOIT, RGPV Bhopal
}

\author{
Sanjeev Sharma \\ SOIT, RGPV Bhopal
}

\begin{abstract}
This paper presents a new face detection method which combines the Skin Color Detector and the Template Matching Method. First we can use the skin Color Detector to find the faces. In the Skin Color Detector, we can use the YCbCr model. The YCbCr model can be used to easily detect the Skin color or Non-Skin color in the images. But in the Skin Color Detector, it can also detect the faces or some non-faces. Finally, we can use the Template Matching Method. This method is used to remove the non-faces and to detect the faces more accurately. Experimental results show that the performance of the proposed method is better than the Skin Color Detector.
\end{abstract}

\section{Keywords}

Edge Detection, Face detection, Skin Color Detector, Template Matching Method.

\section{INTRODUCTION}

Face Detection is a task in images investigation which has many and more applications, such as Facial Expression Analysis, Human Computer Interface (HCI ), Security Systems, FaceRecognitions, Surveillance System, Personal Identity, Verifications, Man-Machine Interface, Content Base Image Retrieval (CBIR) etc. Face Detection is an interesting and challenging problem. Face Detection in pattern recognition technology and computer vision technology as an important subject has high commercial value and an academic value. The main aim of face detection is to determine the location of probable faces in images [1,3]. The comprehensive survey of Face Detection has been given out [2, 4], Skin Color [5, 6], Template Matching Method [1,7]. Face detection is used to determine the size of human faces and locations in the digital images. It can detect facial features and ignores anything else, such as bodies, buildings, and trees etc. Face detection according to various approaches, are classified into four categories such as (i)- Knowledge based method, (ii)- Feature based method, (iii)Template based method, (iv)- Machine learning method [3]. In this paper, we have proposed a new method which is the combination of skin color detector and template matching method. Related work is explained in section II. Proposed work is explained in section III. Experimental results are described in section IV. And finally, conclusions are given in section V.

\section{RELATED WORK}

\subsection{Skin Color Detection}

Color is a powerful fundamental cue of human faces, distribution of skin color clusters lay on a small region of the chromatic color space [3,9]. Skin color can be used in the information of the facial features such as geometry, shape, etc. The skin color detection is an important role in the face detection. It can be used to detect the skin region or non- skin region in the images. First we can choose the suitable color spaces in the skin color detection. There are various color spaces such as, RGB (Red Green Blue), NTSC , YCbCr, HSV, CMY, HIS, YUV, CIE-Lab etc. In the NTSC color space, the main advantages of this color space is that gray-scale information is separate from color data. It can consists the three components such as luminance (Y), hue (I), and saturation (Q). The $\mathrm{YCbCr}$ color space are widely used in the digital images and the digital videos. In the $\mathrm{YCbCr}, \mathrm{Y}$ is the luminance information of the component, and color information is stored as two color difference components, $\mathrm{Cb}$ and $\mathrm{Cr}$. $\mathrm{Cb}$ is the difference between the blue component and a reference value and $\mathrm{Cr}$ is the difference between the red component and a reference value. The HSV (Hue Saturation Value) color space is one of several color systems used by people to select colors. The CMY (Cyan Magenta Yellow) color space. The HSI (Hue Saturation Intensity) color space, it is widely used in skin color detection. YIQ and YUV are analog spaces, while $\mathrm{YCbCr}$ is a digital digital color system [8].

\subsection{Template Matching Method}

Template matching methods [10] that find the similarity between the input images and the template images (training images). Template matching method can use the correlation between the input images and stored standard patterns in the whole face features, to determine the presence of a whole face features. This method can be used for both face detection and face locations. In this method, a standard face (such as frontal) can be used. The advantages of this method are that it is very simple to implement the algorithm, and it is easily to determine the face locations such as nose, eyes, mouth etc based on the correlation values. It can be apply on the various variations of the images such as pose, scale, and shape. Sub-templates, Multiresolutions, and Multi-scales have been proposed to achieve the shape and the scale invariance. Craw et al. presented a localization method based on a shape template of a frontal view face $[1,7]$. A Sobel filter is used to extract the edges. 


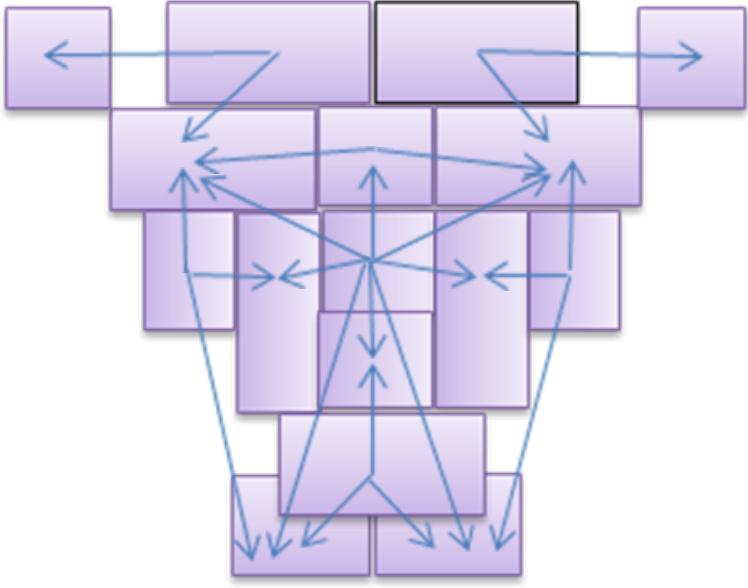

Fig 1: A 14x16 pixel ratio template for face localization, the template is composed of 16 regions (rectangular boxes), and 23 relations ( shown by arrows) [ 1 ]

\section{PROPOSED WORK}

In this paper, we have proposed a new face detection method for color images which uses a combination of the Skin Color Detection and the Template-Matching Method. Fig 2. Shows the structure of the proposed method. In the first stage, we have used the skin color detector. It is used to detect the skin or nonskin regions in the images. In the skin color detector, we have used the $\mathrm{YCbCr}$ color space because it is widely used in the digital images. In the YCbCr, Y channel is represents the luminance component of the images, and both $\mathrm{Cb}$ and $\mathrm{Cr}$ represents the chrominance components of the images. We have then distribution of human skin color modeled with a GMM (Gaussian mixture model) of [11]..

where $\pi_{\mathrm{n}}$ are the mixing parameters, $\mathrm{i}$ is the number of mixture components $(\mathrm{i}=3)$ in our case, and $\mathrm{p}(\mathrm{c} / \mathrm{skin})$ is the Gaussian probability density function can be written as -

$$
\begin{aligned}
& p(c \mid \text { skin })=\sum_{n=1}^{i} \pi_{n} p_{n}(c \mid \text { skin }) \quad \ldots \ldots \ldots \ldots \ldots \\
& p(c \mid \text { skin })=\frac{1}{2 \pi\left|\sum_{\mathrm{s}}\right|^{\frac{1}{2}}} \cdot e^{-\frac{1}{2}\left(c-\mu_{s}\right)^{T} \sum_{s}^{-1}\left(c-\mu_{s}\right)}
\end{aligned}
$$

Here, $\mu_{\mathrm{s}}$ is the mean of vector, $\sum_{s}$ is a covariance matrix, and $\mathrm{c}$ is a color vector.

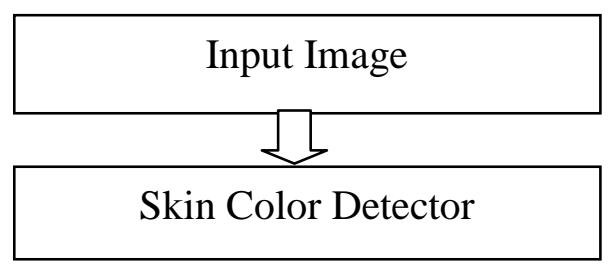

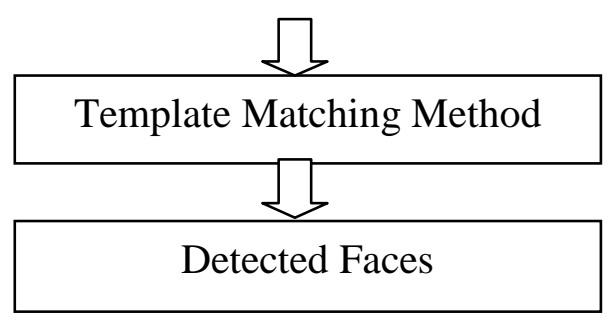

Fig 2: The structure of the proposed method

In this fig 2. First we have applied the input images. All input images are transfered in the skin color detector. The skin color detector have to detect the skin regions or non-skin regions. Skin regions have consists the faces or non-faces, while non-skin regions also consists only the non-faces. So we have to remove the non-skin regions. Then we have selected the skin regions. The disadvantages of the skin color detector is that it also detects non-face regions in addition to faces because it only detects skin pixels. The result of the skin color detector (faces or non-faces) is applied to the template matching method. Finally, the template matching method is used to detect the more accurate faces, and neglect the non-faces. In the template matching, we have used the trained images (eigenfaces). We can easily find the position of the eyes, nose, mouth, eyebrows, head etc. by the use of template matching method. Therefore, we have detect the faces in this method. The block diagram of skin color detector is shown in fig. 3

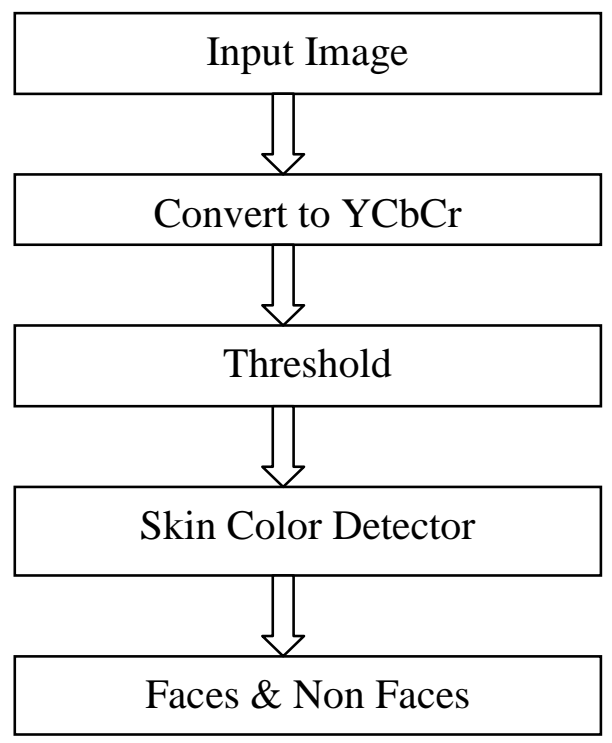

Fig 3: Block diagram of skin color detector

In the template matching method, the template matching is the part of the segmentation of images. In the image segmentation, there are various methods such as template matching, edge detection, clustering, boundary detection, thresholding, texture matching. But in the proposed method, we have used only edge detection and template-matching. The sobel edge detector is used to detect the edges. The trained images are used in the template matching, so we can easily find the location of eyes, nose, mouth etc. then we can detect the faces and remove the 
non-faces. The block diagram of the template matching is shown in fig. 4

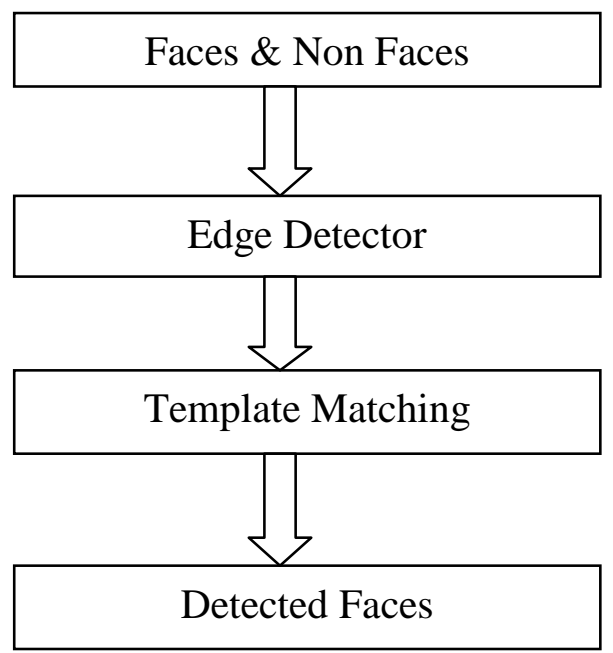

Fig 4: Block diagram of template matching method

\section{EXPERIMENTAL RESULT}

The experiments of the face detection system are carried out on Window XP operating system, and on a $2.2 \mathrm{GHz}$ processor with 1 GB of RAM. It is implemented by a MATLAB version R2007b. We have created the database named RGPV. This database can consists of 30 images. These images contains multiple faces with variations in color, scale, size, expression and positions etc. In this method first we have applied the input image as shown in fig .5 we have converted the RGB image to the gray-scale image, then we can convert the gray-scale image to the $\mathrm{YCbCr}$ image. So we can apply the threshold and convert the threshold image to the binary image. In this image, the value of the skin region is 1 (white), and the value of the non-skin region is 0 (black). Next we have applied the skin color detector, it can detect the next skin regions (skin pixels) and remove the non-skin pixels. But in this method we can also detect some non-faces. So we have applied the sobel edge detector and finally we have applied the template matching, and then produced the output results of the face detection. The template matching method has detected faces more accurately, as shown in fig. 5 shows the steps of the proposed method. It is clear from the results, that proposed method has detected very few non faces than the skin color detector. Thus, we have improved the face detection process by reducing the false positives (non faces detection). The improvement is done by using template matching method in addition to skin color detector. The performance of the proposed method as shown in Table 1, In this table we have compare the Skin color detection method and the proposed method (combined the skin color detector and template matching method).

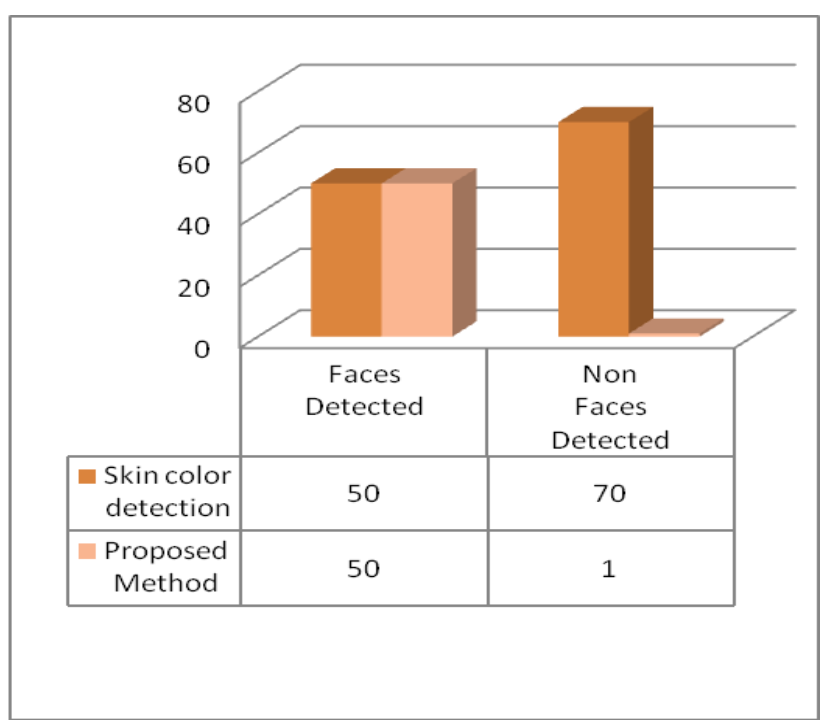

Fig 5: Performance of the Proposed Method
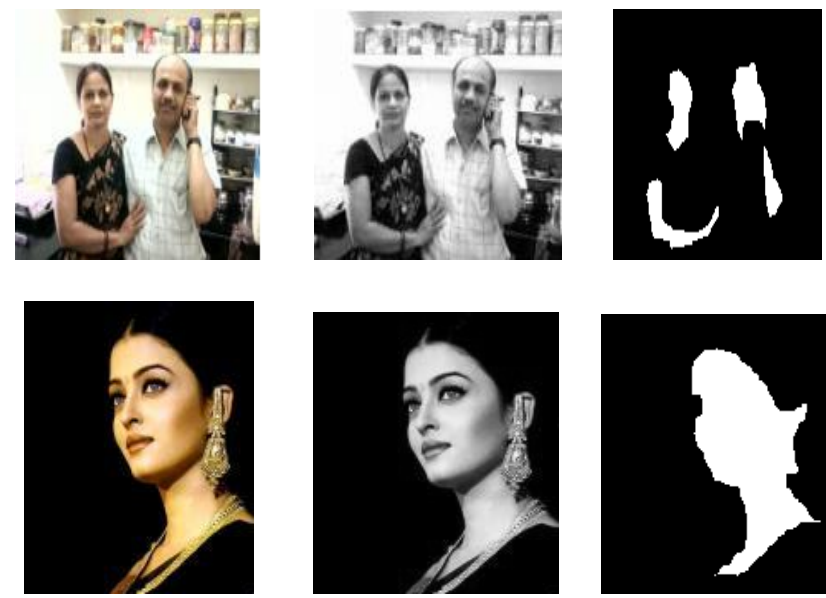

Input Images
Gray-scale Images

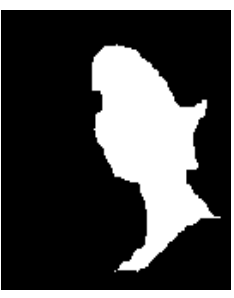

Binary Images
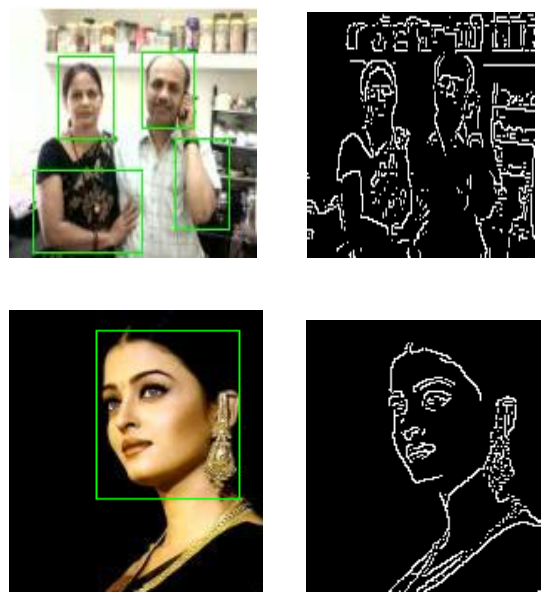

Result of skin color detector

Fig 6: The steps of the proposed method
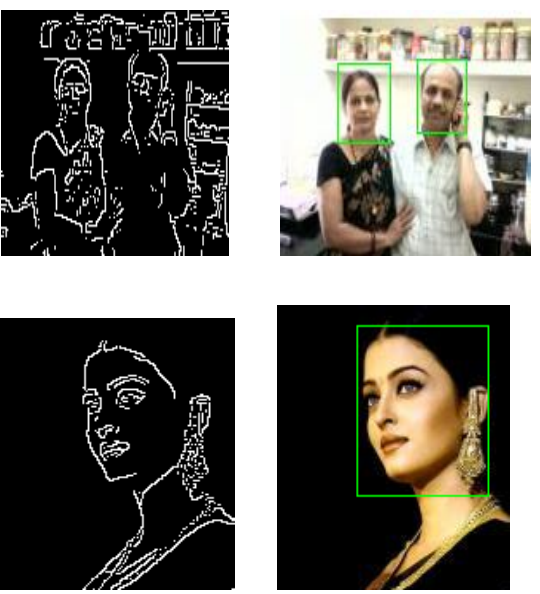

Sobel edge detection

Output 


\section{CONCLUSION}

In this paper, an efficient face detection method using combined skin color detector and the template matching method. The skin color detector is to detect the skin pixels, and also detect the faces or non-faces in the input images. To reduce the non-faces in the detection, we have used the skin color data. And finally, we have applied the template-matching method. The proposed method is compared with the skin color detector. In future we are going to use one or more color spaces in the skin color detector. Also we can find which combination of color spaces gives the best performance.

\section{REFERENCES}

[1] M.Yang, D.J Kriegman, N. Ahuja, January 2002. Detecting Faces in Images : A Survey. In IEEE Transactions on Pattern Analysis And Machine Intelligence.

[2] Paul Viola, Michael Jones, 2001 Rapid Object Detection using a Boosted Cascade of Simple Features, Proceedings IEEE Conf. On Computer Vision and Pattern Recognition, Kauai, Hawaii, USA.

[3] Sania Arjomand Inalou, Shohreh Kasaei, 2010. AdaBoostbased Face Detection in Color Images with Low False Alarm, In IEEE Transaction on 2010 Second International Conference on Computer Modelling and Simulation.

[4] E. Hjelmas, and B. K. Low, Sep2001. Face Detection : a survey, Computer Vision and Image Understanding.

[5] Wang Zhanjie, , Tong Li, Shwu-Huey, and Yang-Takao, 2008. A Face Detection Based on Skin Color and Neural
Network, In IEEE Transaction on International Conference on Computer Science and Software Engineering.

[6] V. Vezhnevets, V. Sazonov, and A. Andreeva, 2003. A Survey on pixel-based skin color detection techniques, In Proc. Graphicon.

[7] Y. Hori, K. Shimizu, Y. Nakamura, and T. Kuroda, 2004. A Real-Time Multi Face Detection Technique Using Positive-Negative Lines-of-Face Template, In Proceedings of the $17^{\text {th }}$ International Conference on Pattern Recognition (ICPR'04), Vol.1, pp. 765 - 768.

[8] Douglas Chai, And A. Bouzerdoum, 2001, A Bayesian Approach to Skin Color Classification in YcbCr Color Space, In Proc. Of British Machine Vision Conference, Vol. 2, pp. 491-500.

[9] C. P. Papageorgion, M. Oren, T. Poggio, 1998, A General Framework for Object Detection, International Conference on Computer Vision.

[10] I. Craw, H. Ellis, and J. Lishman, 1987. Automatic Extraction of Face Features, Pattern Recognition Letters, Vol.5, pp 183-187.

[11] M. J. Seow, D. Valaparla, and V. K. Asari, 2003. Neural Network based Skin Color Model for Face Detection, Proc. Of the $32^{\text {nd }}$ Workshop on Applied Image Pattern Recognition. 